\title{
DISEÑO DE UN MODELO PEDAGÓGICO PARA LA ENSEÑANZA DE FUNDAMENTOS DE PROGRAMACIÓN DE COMPUTADORES BASADO EN EL USO DE LA TECNOLOGÍA COMO MEDIACIÓN PEDAGÓGICA
}

Por:

Lesley Fabiola Bohórquez Chacón', Yegny Karina Amaya Torrado ${ }^{2}$

\section{RESUMEN}

Al interior de la Universidad Francisco de Paula Santander sede Cúcuta, Colombia, se vienen gestando procesos para el desarrollo de proyectos de investigación que den solución a necesidades identificadas en la población estudiantil nortesantandereana. En este contexto, el Grupo de Investigación y Desarrollo de Ingeniería de Software se encuentra desarrollando un proyecto que será utilizado por la población académica de las Facultades de Ingeniería de las Universidades de la ciudad de Cúcuta; el cual esta orientado a solucionar un problema concreto "Los estudiantes matriculados en cursos que involucran en su contenido temáticas y prácticas relacionadas con el aprendizaje de Fundamentos de Programación de Computadores, reflejan problemas de bajo rendimiento durante el período académico"[1]. El proyecto denominado "Diseño de un modelo pedagógico para la enseñanza de Fundamentos de Programación de Computadores basado en el uso de la tecnología como mediación pedagógica", es una experiencia que enmarca aspectos importantes del diseño de un proceso de enseñanza aprendizaje eficiente para dar solución a una necesidad educativa.

\section{ABSTRACT}

In the interior of the University Francisco de Paula Santander Cúcuta, Colombia, it is developing processes for the development from investigation projects that give solution to necessities identified in the nortesantandereana student population. In this context, the Group of Investigation and Development of Software engineering is developing a project that will be used by the academic population of the Faculties of Engineering of the Universities of the city of Cúcuta; which one is oriented to solve to a concrete problem "the students registered in courses that they involve in his content thematic and practical related to the learning of Fundaments of Computers Programming, reflects problems of low yield during the period académico"[1]. The project denominated "Design of a pedagogical model for the education of foundaments of computers programming based on the use of the technology like pedagogical mediations", is a experience that frames aspects important of the instruccionales enviroment generation efficient to give solution to an educative necessity.

Palabras Claves: Teorías de Aprendizaje, razonamiento abstracto, modelación analítica y esquematización, comprensión de lectura y raciocinio.

PACS: 01.40.Gm; 01.50.Fr; 01.50. $\mathrm{Ht} ; 07.05 . \mathrm{Bx}$.

\section{INTRODUCCIÓN}

La responsabilidad social de las universidades con respecto a la calidad de la educación, les exige apoyar procesos de investigación frente a la solución de necesidades educativas, integrando en un trabajo de colaboración conjunta, su recurso humano, económico y de información. Las necesidades educativas detectadas en diferentes profesiones pueden ser caracterizadas por elementos comunes con respecto al área o región del conocimiento que las enmarca. En tal sentido un gran sector de la población estudiantil de Norte de Santander, inscritos en los programas académicos de diferentes ingenierías presentan

\footnotetext{
' Ingeniero de Sistemas, docente, integrante Grupo de Investigación y Desarrollo de Ingeniería del Software "GIDIS" - Universidad Francisco de Paula Santander, bfabiola@bari.ufps.edu.co

2 Ingeniero de Sistemas, joven investigador, integrante Grupo de Investigación y Desarrollo de Ingeniería del Software "GIDIS" -

Universidad Francisco de Paula Santander, ykamaya@bari.ufps.edu.co
} 
Diseño de un modelo pedagógico para la enseñanza de fundamentos de programación de computadores basado en el uso de la tecnología como mediación pedagógica

debilidades en el aprendizaje de los cursos de Fundamentos de Programación de Computadores [1]; que de acuerdo con el área de conocimiento que los enmarcan, se caracteriza por la falta de habilidad, eficiencia y eficacia en la resolución de problemas, capacidad para dar respuestas acertadas, comprensión verbal e interpretación de lectura.

El Grupo de Investigación y Desarrollo de Ingeniería de Software "GIDIS" en la Línea Informática Educativa, miembro de una comunidad universitaria y atentos en compartir la responsabilidad social de la universidad frente a la calidad de la educación y a la sociedad del conocimiento deciden adaptar herramientas informáticas [2] que apoyen en la generación de ambientes instruccionales efectivos.

\section{PROCEDIMIENTO}

Teniendo en cuenta la extensión de esta investigación el grupo orienta la formulación del proyecto por medio de la siguiente hipótesis:

"Los Estudiantes que se apoyan en el uso de herramientas informáticas para realizar el proceso de aprendizaje de Fundamentos de Programación de Computadores evidencian mejor rendimiento durante el período académico, que aquellos que no las usan." [1]

Para comprobar la hipótesis planteada, el proyecto se dividió en las siguientes etapas: Primera Ełapa. Identificación de las causas que generan problemas en el aprendizaje de fundamentos de programación de computadores (Terminada); Segunda Etapa. Diseño de la solución (Terminada); Tercera Etapa. Implementación de la solución planteada (En desarrollo); Cuarta Etapa. Validación de la solución planteada (En desarrollo); Quinta Etapa. Comprobación de la hipótesis planteada (En formulación).

Considerando que la primera etapa del proyecto denominada Identificación de las Causas que generan problemas en el aprendizaje de Fundamentos de Programación de Computadores [1] ya está terminada es necesario mencionar los instrumentos que se utilizaron y a quienes se les aplicaron:

- Para el caso de los estudiantes, quienes fueron parte fundamental del proceso, se les aplicó una encuesta y un cuestionario de habilidades mentales en las universidades: Fundación Universitaria San Martín, Corporación Universitaria de Santander, Universidad Simón Bolívar y Universidad Francisco de Paula Santander; el total de la población objeto de estudio fue 407 estudiantes, a quienes se les aplicó los instrumentos dado que el tamaño no ameritaba trabajar con una muestra [1].

- Para los 15 docentes considerados facilitadores, mediadores $u$ orientadores en el proceso de aprendizaje del estudiante, se realizó una entrevista para reforzar y complementar la información de los estudiantes; una guía de observación donde se registraron los hechos percibidos durante la clase y se aplicó una técnica de análisis de contenidos curriculares para poder relacionar contenidos con el rendimiento de los estudiantes [1].

Para conocer los formatos de los instrumentos remítase al estudio [1].

Luego de aplicar el cuestionario de habilidades mentales a los estudiantes, tabular y analizar la información recolectada se concluyó:

- El 32\% de la población objeto de estudio se les dificulta resolver problemas basados en deducciones lógicas, hacer planes y ponerlos en ejecución partiendo de los hechos existentes.

El $64 \%$ de los estudiantes encuestados tiene dificultad para entender ideas expresadas en palabras, esencial para todas las actividades en 
Diseño de un modelo pedagógico para la enseñanza de fundamentos de programación de computadores basado en el uso de la tecnología como mediaciones pedagógicas

las cuales la información se obtiene mediante la lectura $u$ oyendo el lenguaje hablado.

- Se identificó que la mitad de los estudiantes presentan deficiencias en lógica, secuenciación y observación fundamentales para obtener la habilidad de visualizar objetos de dos o tres dimensiones, y las relaciones mutuas entre los objetos; presentan inconvenientes para desarrollar problemas lógicos por medio de un enunciado y formular la situación a través de un modelo matemático; además no analizan un pasaje escrito desde todas sus perspectivas para reconocer tanto los elementos explícitos como las inferencias y conclusiones que se sugieren.

- Por lo tanto se puede concluir que los estudiantes tienen deficiencias en las habilidades mentales necesarias para el desarrollo del pensamiento complejo (las habilidades para razonar, tomar decisiones, resolver problemas y afrontar situaciones de la vida cotidiana, académica y profesional).

De acuerdo al resultado del primer estudio se concluye que el proyecto debe implementar un modelo pedagógico que permita atacar las falencias encontradas en las habilidades de Razonamiento Abstracto, Modelación Analítica y Esquematización, Raciocinio, Comprensión Verbal, Comprensión e Interpretación de Lectura; y se propone una solución que gira en torno a dos intereses fundamentales: Potenciar las habilidades mentales que promueven el desarrollo de un pensamiento complejo y el uso de estrategias pedagógicas que mejoran la eficacia del proceso de enseñanza - aprendizaje, potenciando la actividad del educando, la interacción con el docente y con sus pares y la comprensión de los contenidos curriculares desde una concepción que parte del constructivismo pero no se circunscribe únicamente a él.

El grupo de investigación para el desarrollo de la segunda etapa del proyecto "Diseño de la
Solución", diseña un proceso de aprendizaje el cual permite dar respuesta a seis grandes preguntas a saber [8]:

1) ¿̇En qué deben contribuir estas asignaturas al proceso de formación del Ingeniero?

- Al esfuerzo del sentido de la responsabilidad, la autonomía, el liderazgo, el trabajo en equipo y al autoaprendizaje del ingeniero.

- A ampliar las capacidades intelectuales de análisis, comparación, modelación, cálculo, graficación, deducción.

- A enriquecer el campo perceptual del ingeniero y las operaciones de procesamiento de la información.

- Propiciar el descubrimiento de principios y reglas lógicas de inferencia y deducción. De esta forma se aprenden conceptos básicos que pueden ser transferidos a situaciones nuevas.

- A desarrollar destrezas en la Planificación de estrategias para la resolución de problemas y en la localización de información definida dentro de una gran masa de conocimientos, mediante el desarrollo de algoritmos.

2) ¿Qué estrategias utilizar para el logro del Aprendizaje?

- Diagnóstico previo de habilidades mentales primarias: Comprensión verbal, Comprensión e interpretación de lectura, Razonamiento abstracto, Modelación Analítica y esquematización y Raciocinio.

- Refuerzo individual de habilidades mentales primarias.

- Desarrollo de contenidos.

- Ejercitación y práctica en el desarrollo de problemas reales.

- Monitoreo del desarrollo de habilidades Vs logros de aprendizaje.

- Seguimiento y evaluación. 
Diseño de un modelo pedagógico para la enseñanza de fundamentos de programación de computadores basado en el uso de la tecnología como mediación pedagógica

3) ¿Qué medios utilizar para el desarrollo de la estrategia?

- En el Diagnóstico. Se sistematiza a través de los niveles básicos de una herramienta computacional (software) para el desarrollo de habilidades mentales. La computadora en si misma no constituye la herramienta de diagnóstico, por tanto debe ser administrada y consultada por el docente a cargo de la asignatura. El diagnostico previo también es susceptible de desarrollarse mediante talleres impresos por la herramienta.

- Refuerzo individual. Se sistematiza a través de los niveles principiante, intermedio y avanzado de cada software.

- Desarrollo de contenidos. Se lleva a cabo en el aula de clase, mediante la utilización de recursos bibliográficos, talleres, clases magistrales.

- Ejercitación y práctica en el desarrollo de problemas reales. Se sistematiza mediante el uso de un Módulo de Ejercitación y Práctica. Diseñado de acuerdo a los siguientes criterios: Planteamiento de situaciones y soluciones, desarrollo del pensamiento lógico, incremento de la capacidad creativa y de investigación, enfoque interdisciplinario que permita al estudiante la adquisición integración y aplicación de conocimientos.

- Monitoreo del desarrollo de habilidades. Se sistematiza mediante el almacenamiento de los resultados de las evaluaciones sumativas que ofrece la herramienta. Mediante el monitoreo se registran estados reales del desarrollo alcanzado por el estudiante en cada habilidad y es el docente quien a través del Módulo Administrador de cada Herramienta, captura los elementos básicos para hacer el seguimiento.

- Seguimiento. Se logra mediante un diálogo proactivo Docente - Estudiante - Grupo de estudiantes y viceversa, construyendo pactos de cualificación a través de los elementos básicos provenientes del monitoreo, aliviando la tensión normal entre el debe ser (ideal) del plan diseñado y el ser (real) de la ejecución de los refuerzos y prácticas individuales.

- Evaluación. Como fase complementaria comprende también dos tareas centrales: análisis y elaboración de juicios por parte del docente. Se sistematizan las evaluaciones formativas, que se realizan al finalizar cada tarea de aprendizaje. Estas evaluaciones se pueden diseñar mediante el Módulo administrador de la Herramienta de Ejercitación y Práctica, ser impresos o aplicados mediante la misma herramienta.

4) ¿̇Qué insumos son relevantes para la toma de decisiones, durante el proceso de aprendizaje?

El docente analiza los resultados obtenidos de la evaluación. La tarea del análisis implica el reconocimiento de singularidades a través de eventos comunes, presentes en los resultados de diferentes estudiantes, para conformar grupos, con el fin de emitir juicios. La emisión de juicios integra en forma de indicadores globales o proposiciones el estado de cada componente básico (habilidad) y del logro de aprendizaje en general y con frecuencia se debe regresar a dialogar con la fase de análisis. La comunicación entre estas dos fases permite sintetizar y emitir juicios de valor que expresan las cualidades presentes tanto en el estudiante como en la estructura global del aprendizaje. También los juicios permiten organizar y proponer nuevas estrategias para aproximar aún mas los valores de los indicadores a los deseables e incluso superarlos.

El resultado de la fase de evaluación, los juicios de valor, se constituyen en el insumo vital para la dirección del proceso de aprendizaje por parte del docente puesto que a través de estos puede adelantar su tarea principal tomar decisiones. En este punto en el que dialogan, la fase de evaluación y la dirección del proceso se configura una transición crítica entre la terminación de un ciclo y la posible iniciación de otro igual o sujeto a cambios y ajustes desde pequeños hasta fundamentales. 
Diseño de un modelo pedagógico para la enseñanza de fundamentos de programación de computadores basado en el uso de la tecnología como mediación pedagógica

5) ¿Qué criterios utilizar para la organización y el desarrollo de Contenidos?

- Dimensión de los Fundamentos de Programación de Computadores.

- Concepto de competencias (Descubrimiento, Interactividad Visual y Auditiva, Desarrollo Individual, Enseñanza Guiada, Participación, Trascendencia).

6) ¿̇Cuáles deben ser los aspectos propios de la labor docente y de la labor de los estudiantes, los atributos e indicadores típicos del logro de aprendizaje?

Los aspectos propios tanto de la labor docente como el estudiante se definen en su comportamiento tanto en el aula de clase, como en su espacio individual de trabajo y están dados por los siguientes atributos: Trascendencia, Proposición, Persuasión, Profundidad, Visión, Argumentación, Interrogación, Cumplimiento, Aceptación o Impacto, Liderazgo, Responsabilidad, Autonomía y Clima de Aula.

La metodología utilizada responde a modelos de desarrollo de currículos integrados, y se diseña con la experiencia docente de los investigadores, la consulta y observación de metodologías pedagógicas establecidos mediante el estudio, y se nutre de fuentes secundarias como internet, material bibliográfico, artículos y ponencias. También cabe resaltar que no se pretende asumir de manera sistemática y sistémica el modelo de aprendizaje que aquí se propone, puesto que dada la complejidad de la naturaleza del trabajo de aula, las fases de Evaluación y seguimiento posibilitan asumir el proceso como un medio de investigación para la creación de métodos heuristicos para el logro de un aprendizaje exitoso en el área.

Para el desarrollo de la tercera etapa "Implementación de la solución planteada" se trabajo con una metodología establecida por el grupo para el
Desarrollo de Software Educativo [3] la cual reúne aspectos de:

1. Metodología de Álvaro Galvis Panqueva [4]

2. Metodología ISE_OO [5]

3. Metodología de Guiones

4. Bases Epistemológicas de Pedagogía

5. Procesos de Análisis y Diseño de Ingeniería del Software [6]

6. Principios de Aprendizaje para selección y uso de medios de instrucción. [7]

Con el grupo de trabajo se concluyó que el proyecto debía dividirse en 5 módulos ya que los resultados arrojados por el cuestionario que se les aplicó a los estudiantes fue bajo, demostrando las falencias en: Razonamiento Abstracto, Modelación Analítica $Y$ esquematización, Raciocinio, Comprensión Verbal, Comprensión e Interpretación de Lectura.

El grupo de trabajo decide que aparte de los cinco módulos mencionados anteriormente, debe haber un sexto módulo de ejercitación donde el estudiante solo trabajará con ejercicios lógicos donde se podrá analizar si el estudiante tiene las habilidades necesarias para el desarrollo del pensamiento complejo. Los cinco primeros módulos se desarrollaron como Sistemas Tutoriales [4] porque son los que más se adaptan a la integración de Tecnologías de información en procesos de Aprendizaje, además con este software se quiere que el docente asuma el papel de un buen tutor, guiando al aprendiz a través de las distintas fases del aprendizaje, mediante una relación dialogal. Típicamente un tutorial incluye las cuatro grandes fases que según Gagné [7] deben formar parte de todo proceso de enseñanza-aprendizaje: introductoria, orientación inicial, aplicación y retro-alimentación.

El proyecto enmarca el diseño de los medios de Seis módulos que a su vez están conformados por dos submódulos (Usuario y Administrador); en los cuales el usuario puede interactuar con las diferentes 
Diseño de un modelo pedagógico para la enseñanza de fundamentos de programación de computadores basado en el uso de la tecnología como mediación pedagógica

opciones que conforman cada uno de los módulos, "Introducción" en esta fase el estudiante encuentra el contenido de los temas a tratar en cada uno de los módulos; "Orientación" en la cual el estudiante encuentra un video de la forma de interactuar con el software; "Ejercitación" en esta fase el estudiante puede resolver una serie de ejercicios e ir conociendo el puntaje que obtiene de sus respuestas correctas e incorrectas; también puede realizar una evaluación general; el administrador tiene almacenado en la base de datos un registro de usuarios, de cuantas sesiones ha ingresado el usuario al software y el respectivo puntaje obtenido en la evaluación general.

En el módulo de ejercitación se utilizó la metodología ISE-OO (Ingeniería de Software Educativo - Orientado a Objetos) [5]; ya que el desarrollo de este Software Educativo implicaba seguir una metodología que ofreciera una manera clara y sólida de cómo integrar el aspecto educativo e informático.

\section{RESULTADOS}

- Modelo pedagógico para la enseñanza de Fundamentos de Programación de Computadores basado en el uso de la tecnología como mediación pedagógica.

- Esquema de aprendizaje propuesto por los integrantes del grupo de investigación:

La Figura 1. Corresponde al esquema del Proceso de Aprendizaje, se puede apreciar que el proceso comienza con el diagnostico previo realizado con una prueba que se le hace al usuario antes de comenzar a trabajar con la herramienta, con el fin de conocer en el nivel que se encuentra. Luego de interactuar con los niveles que tienen los módulos y a su vez desarrollar los contenidos del curso; se analizan los resultados obtenidos en las pruebas que proporciona la herramienta y las que se realiza en el transcurso del curso.

Figura 1. Esquema del Proceso de Aprendizaje

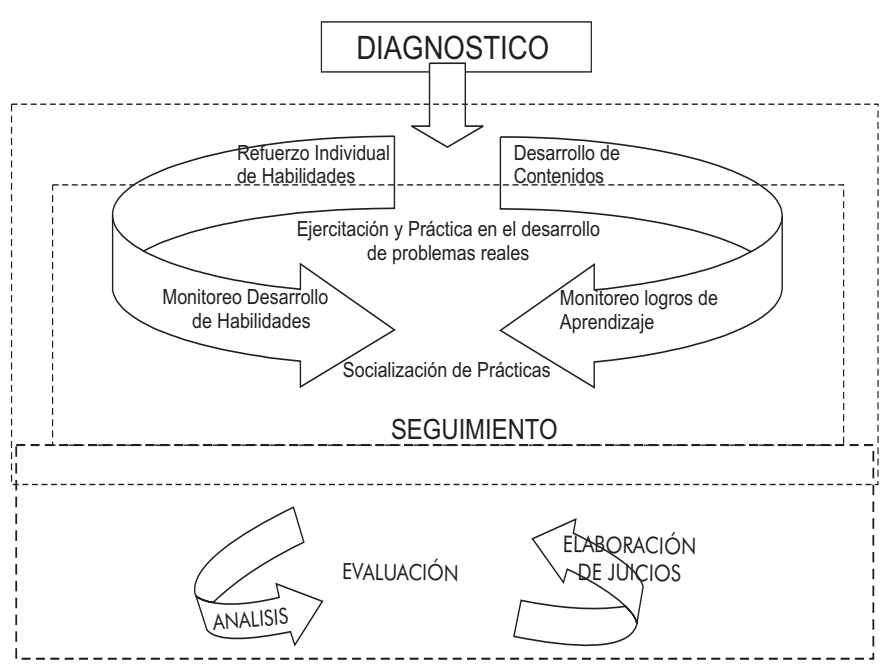

- Diseño del esquema general del proyecto Modelo pedagógico para la enseñanza de Fundamentos de Programación de Computadores basado en el uso de la tecnología como mediaciones pedagógicas, realizado por el grupo de investigación:

La Figura 2. Representa el esquema de la herramienta informática propuesta para el refuerzo individual de habilidades, ejercicios y prácticas. Se observa dos módulos los cuales serán manipulados por los usuarios (Docente - estudiante); una base de datos la cual almacena los datos personales de los usuarios, los registros de sesiones, el puntaje obtenido en cada prueba; las diferentes fases del modulo estudiante (Introductoria, orientación Inicial, Aplicación y Retroalimentación); las interfaces que tiene cada modulo (Razonamiento Abstracto, Modelación Analítica y esquematización, Raciocinio, Comprensión Verbal, comprensión e Interpretación de Lectura y ejercitación) en los cuales pueden acceder los usuarios y los niveles (Principiante, Intermedio y Avanzado). 
Diseño de un modelo pedagógico para la enseñanza de fundamentos de programación de computadores basado en el uso de la tecnología como mediación pedagógica

Figura 2. Esquema de la herramienta informática propuesta para el refuerzo individual de habilidades, ejercicios y prácticas.

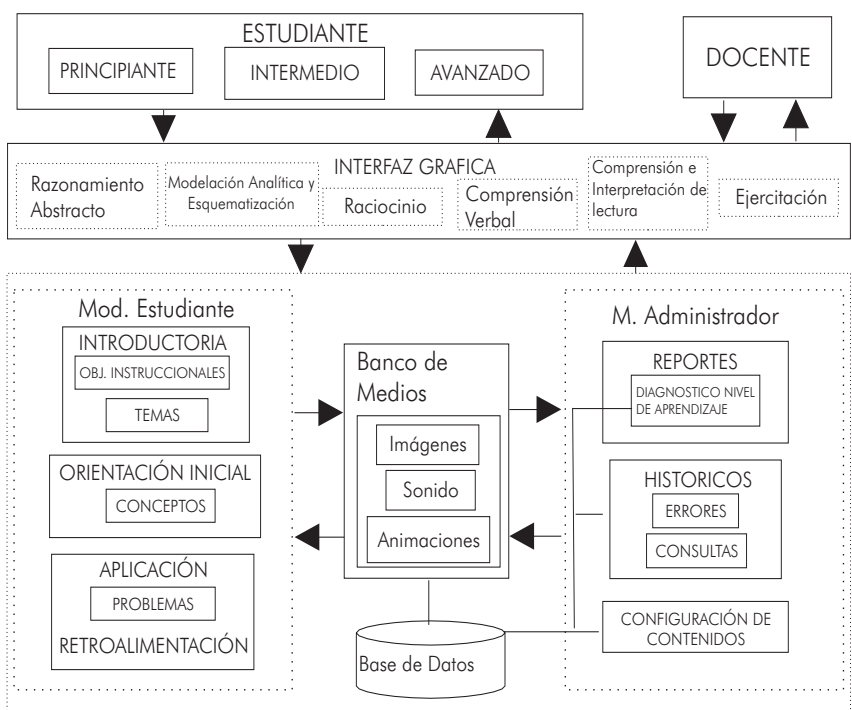

- El resultado que se lleva hasta el momento se observa en la Figura 3. que representa los escenarios principales de los módulos que están terminados y los que están en la etapa de construcción, en los cuales el estudiante puede acceder a los diferentes botones y accesos que tiene cada uno de los módulos.

Figura 3. Escenarios principales de los módulos.
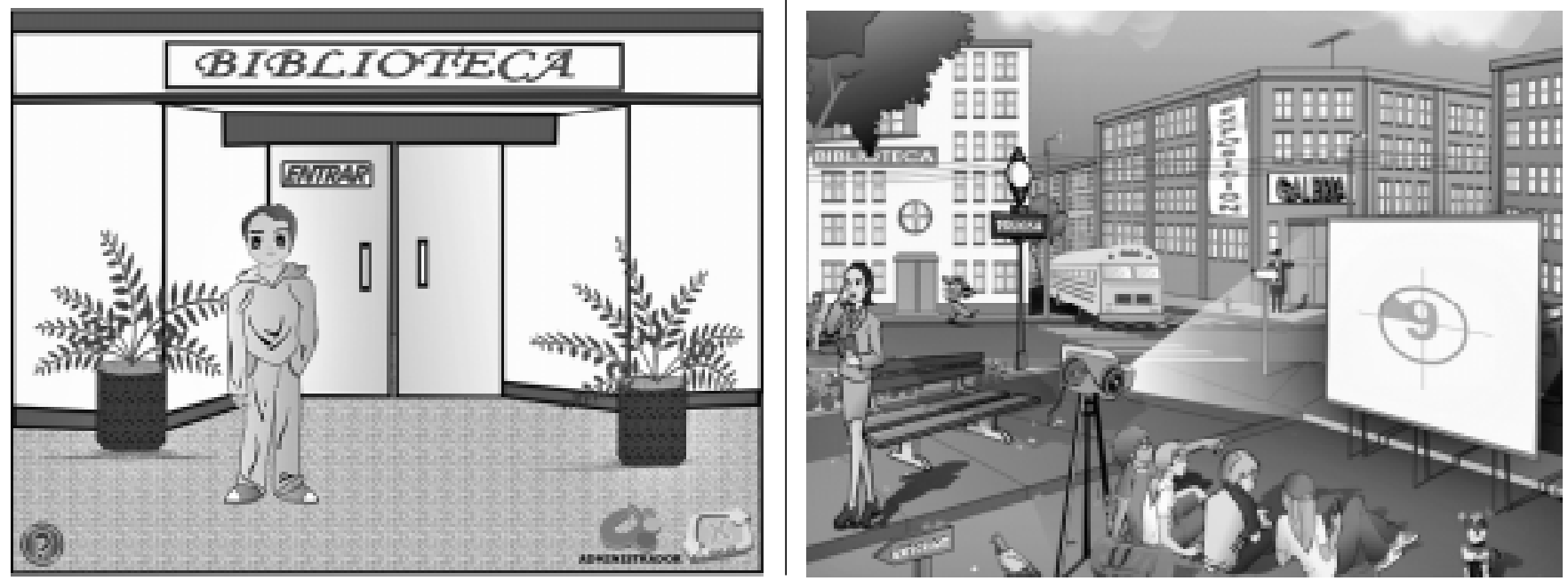

Revista Respuestas - Universidad Francisco de Paula Santander Año 10 No. 1 
Diseño de un modelo pedagógico para la enseñanza de fundamentos de programación de computadores basado en el uso de la tecnología como mediación pedagógica

\section{CONCLUSIONES}

Los cursos que involucran en sus contenidos Fundamentos de Programación de Computadores requieren desarrollar durante el proceso de aprendizaje las habilidades mentales primarias.

La planeación y diseño de un ambiente instruccional contextualizado, requiere de un análisis riguroso de debilidades detectadas tanto en el docente como en el estudiante y la asesoría de un equipo interdisciplinario en la configuración de un modelo de aprendizaje.

De acuerdo a la naturaleza del conocimiento que se pretende construir mediante el proceso de aprendizaje de Fundamentos de Programación de Computadores, es necesario no propiciar ambientes pasivos, por el contrario fortalecer habilidades, valores y competencias entre las cuales se recomienda: proposición, argumentación, visión, interrogación, cumplimiento, liderazgo y responsabilidad.

Se considera que el sistema tutorial y ejercitación y práctica sugeridos en la clasificación realizada por Álvaro Galvis Panqueva son los que más se adaptan a la integración de Tecnologías de información en procesos de Aprendizaje, ya que este software es un proceso complejo que a diferencia de otros tipos de software requiere especial atención, el docente es un guía para el estudiante en el desarrollo de las habilidades para razonar, tomar decisiones, resolver problemas y afrontar situaciones de la vida cotidiana.

\section{AGRADECIMIENTOS}

A los integrantes del grupo de investigación, a los estudiantes que participaron en el desarrollo de los diferentes proyectos, a docentes y asesores que brindaron grandes aportes en la recolección y análisis de información, a la Universidad Francisco de Paula Santander a través del Fondo de Investigaciones Universitarias - FINU por la financiación del soffware y a Colciencias por el apoyo mediante el Programa de Jóvenes Investigadores.

\section{BIBLIOGRAFÍA}

[1] Amaya, Y. \& Angarita, L. (2003). Identificación de las Causas que generan problemas en el aprendizaje de Fundamentos de Programación de Computadores. Memoria para optar el Título de Ingeniero de Sistemas, Programa de Ingeniería de Sistemas, Universidad Francisco de Paula Santander, San José de Cúcuta, Colombia.

[2] Rodríguez, A.; Sempere, F.; Tormo, G. \& Pedro, D. Herramienta Informática par ala realización y evaluación de pruebas objetivas (Exámenes Test Multimedia). http://ttt.upv.es/ arodrigu/Testgip/ manuales/TestGIP.pdf

[3] Grupo de Investigación y Desarrollo de Ingeniería de Software. Universidad Francisco de Paula Santander. Metodología para el Desarrollo de Software Educativo. (En impresión).

[4] GALVIS, A.H. (1986). Ingeniería de Software Educativo. Bogotá: Universidad de los Andes, Departamento de Sistemas y Computación.

[5] GALVIS PANQUEVA, Álvaro, Ingeniería de Software Educativo con modelaje orientado por objetos; un medio para desarrollar Micromundos Interactivos, Vol. $11 \mathrm{~N}^{\circ} 1,1998$, pp. 9 -30.

[6] PRESSMAN, Roger S. Ingeniería del Software. Cuarta Edición. Madrid: McGraw- Hill, 1997. p. 118.

[7] GAGNÉ R. M. (1974). Principios de Aprendizaje para Selección y Uso de Medios de Instrucción. En A.H. Galvis (1987). Ingeniería de Soffware Educativo parte 2. Bogotá: Universidad de Los Andes (77-119).

[8] Bohórquez, L. Diseño de una solución para el proceso de aprendizaje de Fundamentos de Programación de Computadores. (En impresión).

Recibido: Febrero 7 de 2005

Aceptado: Junio 27 de 2005 